\title{
Discrete element model for quasi-brittle rupture under tensile and compressive loading
}

\author{
Georg Koval ${ }^{* \dagger} \dagger$, Ba Danh Le and Cyrille Chazallon \\ ICUBE Laboratory, UMR 7357, CNRS, National Institute of Applied Sciences of Strasbourg, 24 Boulevard de la \\ Victoire, 67084 Strasbourg, France
}

\begin{abstract}
SUMMARY
In the absence of initial cracks, the material behavior is limited by its strength, usually defined in homogeneous conditions (of stress and strain). Beyond this limit, in quasi-brittle case, cracks may propagate and the material behavior tends to be well described by fracture mechanics. Discrete element approaches show consistent results dealing with this transition during rupture. However, the calibration of the parameters of the numerical models (i.e., stiffness, strength, and toughness) may be quite complex and sometimes only approximative. Based on a brittle rupture criterion, we analyze the biaxial response of uncracked samples. Thus, tensile and compressive strengths are analytically identified and become direct parameters of our discrete model. Furthermore, a physically reliable crack initiation (and subsequent propagation) is shown to be induced during rupture and verified by the simulation of three-point bending and diametral compression tests. Copyright @ 2016 John Wiley \& Sons, Ltd.
\end{abstract}

Received 12 November 2015; Revised 14 March 2016; Accepted 22 March 2016

KEY WORDS: quasi-brittle rupture; discrete element method; crack initiation; tensile and compressive strengths

\section{INTRODUCTION}

Fracture mechanics is very useful to describe the behavior of structural components presenting cracks and other imperfections, provided that each defect is precisely defined on the models. The performance of uncracked elements mostly depends on countless microscopic defects that are intrinsic to each material. In quasi-brittle materials, we usually define strength as the effort leading to the propagation of these inner defects that tend to become large cracks. Roughly, we observe a transition from the crack initiation (triggered by strength of the materials) and its propagation (described by fracture mechanics). In order to identify this transition, the notion of crack band (defined by its thickness $h$ ) was proposed [1-3]; high-stress gradients (at the vicinity of a crack, for example) induce small values of $h$, whereas in homogeneous stress, $h$ contain all the sample (Figure 1). When $h$ is small, fracture mechanics can be applied; the material behavior depends on its toughness. When $h$ is large, the individual analysis of each small defect is not viable; the use of failure criteria (Rankine, Mohr-Coulomb, Von Mises, etc.) becomes more practical.

However, the difficulty often lies on the description of intermediate scales of $h$. Let us suppose a pre-cracked sample; if the existing notch may not be sharp enough or large enough to mobilize the material toughness, the failure mechanism may be dependent on the strength of the material. The quasi-brittle behavior under tensile stresses has been treated by many authors [3-6]. Novozhilov [4] propose a simple failure criterion based on the average normal stress along the predicted trajectory of the cracks. Combining the Griffith's energy criterion for a V-notch and a strength criterion, Leguillon [5, 6] and Zhang [3] present theoretical predictions that are consistent with experimental

\footnotetext{
*Correspondence to: Georg Koval, ICUBE Laboratory, UMR 7357, CNRS, National Institute of Applied Sciences of Strasbourg, 24 Boulevard de la Victoire, 67084 Strasbourg, France.

${ }^{\dagger}$ E-mail: georg.koval@insa-strasbourg.fr 

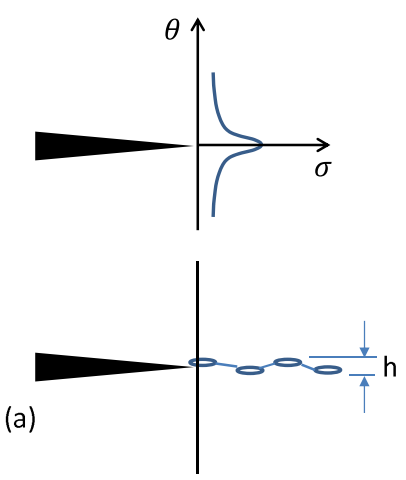

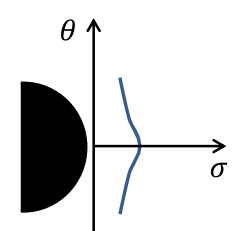

(b)

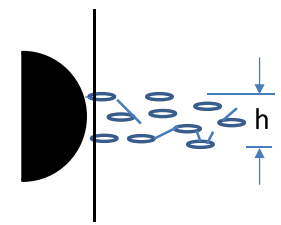

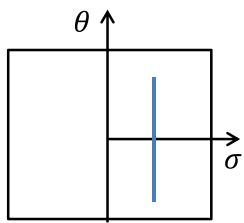

(c)

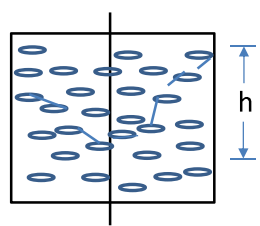

Figure 1. Crack band $h$ according to the stress gradient: (a) under high gradient, near a cracks, (b) under low gradient, near a geometric defect, and (c) under absence of gradient, in uniform stress, modified from the illustration by Zhang and $\mathrm{Li}[3]$.

results. Compressive load may also induce the rupture of quasi-brittle materials by crack propagation, but the mechanism cannot be captured by the previous models, based exclusively on continuum mechanics. A homogeneous material submitted to a uniform compressive stress cannot present tensile stresses that would justify any crack initiation. On the other hand, in discrete element models [7], materials are assimilated to assemblies of particles in contact, which makes the description of this rupture mode physically possible [8-10]. In this approaches, particle geometry and contact parameters control the material behavior. The calibration of failure properties, can be rather complex $[11,12]$ and hide some difficulties, especially related to very different values of compressive and tensile strengths [13], for example. For regular patterns however, analytical expressions for strength can be derived as shown by Liu et al. [14].

In this paper, we present a generalization of the brittle fracture criterion discussed in [15] to the case of homogeneous stress failure. We focus here on the control of the tensile and compressive strengths of uncracked samples. After a rapid description of the discrete model in Section 2, we discuss extensively the biaxial stress behavior in Section 3, where the failure criterion is developed. On the first part of Section 4, we verify numerically the rupture envelope predicted by our equations. On the second part, uncracked samples are simulated (a three-point bending and diametral compression tests) to show the consistency of the initiation of cracks during failure and their subsequent propagation. Finally, we present the conclusions of the work.

\section{DISCRETE ELEMENT APPROACH}

\subsection{Contact model}

The numerical approach is based on discrete element method (DEM) like in $[7,16]$ and is completely described in [15]. At each time step, the computation of all contact forces is followed by the application of Newton's second law to the particles. The motion equations are then solved by Gear's order three predictor-corrector algorithm [17]. Each contact force presents normal and tangential components, respectively, $N$ and $T$ (Figure 2(a)). We can show that an isotropic elastic behavior can be reproduced by the compact arrangement shown on Figure 2(b) $[18,19]$. A representative elementary cell composed by particles of diameter $d$ may be associated to the regular structure of the discrete medium. The contact behavior follows a standard linear spring and dashpot model. We take as value of the damping parameters $c_{n}$ and $c_{t}$ a small fraction of $\sqrt{m k_{n}}$ (where $m$ is the particle mass), which guarantees a negligible inelastic effect. For further analysis, we will focus on the elastic contribution of the contact force, function of the relative displacements $\delta_{n}$ and $\delta_{t}$ (Figure 2(a)).

A direct relation between continuum elastic parameters (Young's modules $E$ and Poisson ratio $v$ ) and discrete elastic parameters (normal and tangential stiffness, $k_{n}$ and $k_{t}$, respectively) can be derived [18], which reads for plane stress: 
(b)

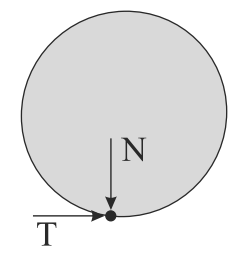

(a)
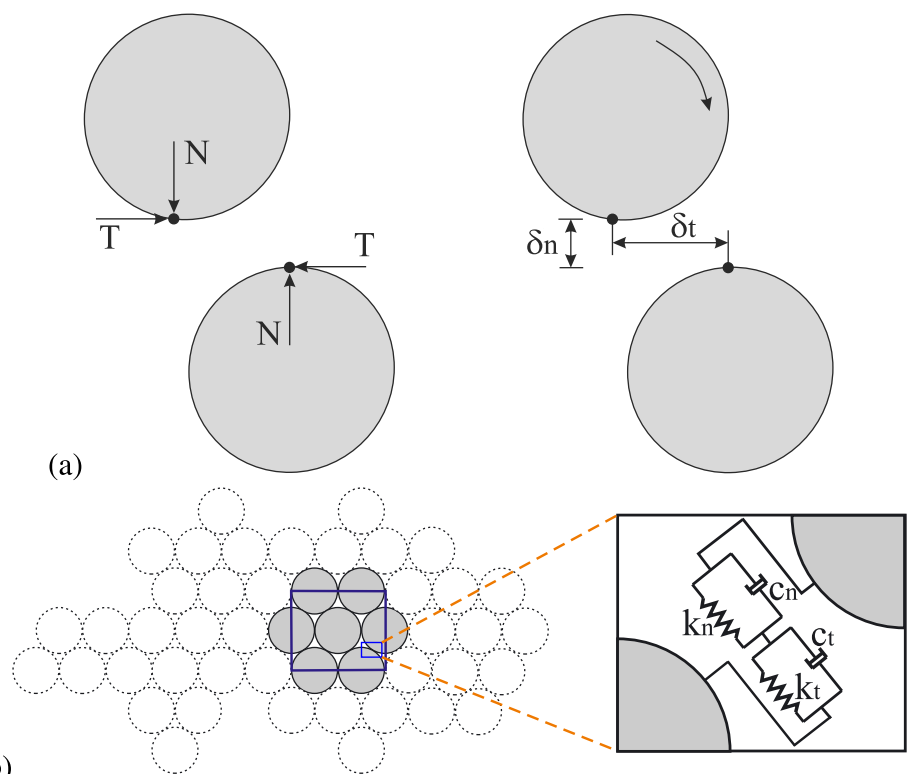

Figure 2. (a) Contact forces and corresponding displacements. (b) Periodic compact arrangement of particles and the contact law.

$$
\left\{\begin{array}{l}
k_{n}=\frac{E}{\sqrt{3}(1-v)} \\
k_{t}=\frac{1-3 v}{1+v} k_{n}=\frac{1-3 v}{\sqrt{3}\left(1-v^{2}\right)} E .
\end{array}\right.
$$

\subsection{Definition of strain and stress in the discrete medium}

2.2.1. Mean strains and mean stresses. The mean values of the components of the tensors of stress and strain are based on the behavior of one pair of contacts $(k i$ and $k j)$ associated to three particles $(i, j$ and $k)$. A local coordinate system $(n ; t)$ is defined, where $t$ virtually connects both contacts for which $n$ is an orthogonal axis (Figure 3(a)). The normal and tangential (relative) displacements associated to contacts $k i$ and $k j$ (respectively, $\delta_{n i k}$ and $\delta_{t i k}, \delta_{n j k}$ and $\delta_{t j k}$, as shown in Figure 3(a)) give rise to mean strain values $\bar{\varepsilon}_{n n}$ and $\bar{\varepsilon}_{t t}$ :

$$
\left\{\begin{array}{l}
\bar{\varepsilon}_{n n}=\left(\delta_{n i k} \sqrt{3}+\delta_{n j k} \sqrt{3}+\delta_{t i k}-\delta_{t j k}\right) /(d \sqrt{3}), \\
\bar{\varepsilon}_{t t}=\left(\delta_{n i k}+\delta_{n j k}-\delta_{t i k} \sqrt{3}+\delta_{t j k} \sqrt{3}\right) / d .
\end{array}\right.
$$

The normal and tangential components of each contact (respectively, $N_{i k}$ and $T_{i k}, N_{j k}$ and $T_{j k}$, as indicated in Figure 3(b)) can be projected over the directions $(n ; t)$ giving rise to resultant forces. Considering the length of a particle diameter $d$, mean stresses (Figure 3(c)) may be associated to these forces:

$$
\left\{\begin{array}{l}
\bar{\sigma}_{n}=\left(N_{i k} \sqrt{3}+T_{i k}+N_{j k} \sqrt{3}-T_{j k}\right) /(2 d), \\
\bar{\sigma}_{t}=\left(-N_{i k}+T_{i k} \sqrt{3}+N_{j k}+T_{j k} \sqrt{3}\right) /(2 d) .
\end{array}\right.
$$

2.2.2. Principal stresses. The stress tensor (in two dimensions) can be defined by the value of the principal stresses $\bar{\sigma}_{I}$ and $\bar{\sigma}_{I I}$ and their orientation. Hence, we define $\beta$ as the angle between 
(a)
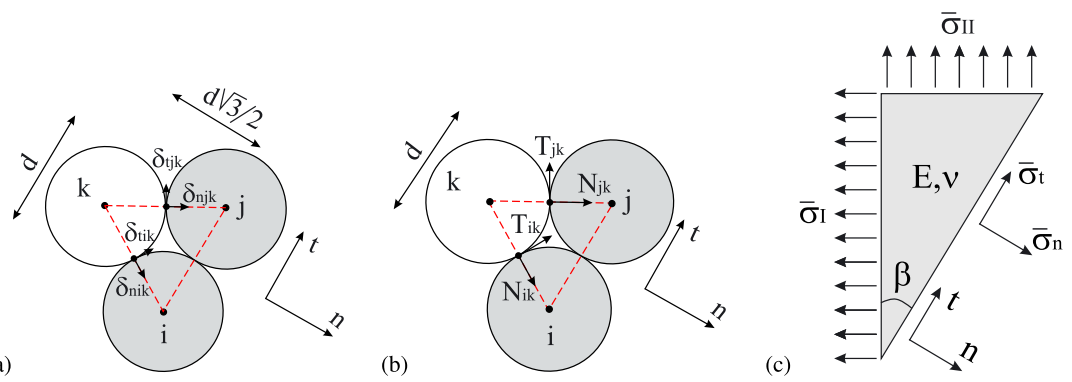

Figure 3. (a) Adjacent particles, respective contact displacements, and (b) contact forces. (c) Mean stresses and the orientation of their principal values.

$(n ; t)$ and the coordinate system associated to the principal stresses (Figure 3(c)). Consequently, the principal stresses may be written as

$$
\left\{\begin{array}{l}
\bar{\sigma}_{I}=\bar{\sigma}_{n}+\bar{\sigma}_{t} \tan (\beta), \\
\bar{\sigma}_{I I}=\bar{\sigma}_{n}-\bar{\sigma}_{t} / \tan (\beta) .
\end{array}\right.
$$

We determine the value of $\beta$ associating the information from mean strains and mean stresses at a pair of contacts (see Appendix A for more details). Hence, the value of $\beta$ can be written as

$$
\beta=-\frac{1}{2} \arctan \left(\frac{2 \bar{\sigma}_{t}}{A}\right)
$$

where $A=\frac{E}{1-v}\left(\bar{\varepsilon}_{n n}+\bar{\varepsilon}_{t t}\right)-2 \bar{\sigma}_{n}$.

\section{BIAXIAL STRESS BEHAVIOR}

In this section, we identify the material strength under biaxial stresses, based on the uniaxial behavior of an uncracked sample. We study a homogeneous stress state generalizing the behavior of the elementary cell, where the contact forces (and displacements) are simply identified. The application of the rupture criterion previously presented in [15] induces naturally a tensile limit to the material. The same idea is then adopted to derive the compressive strength, where physically consistent crack initiation is generated.

\subsection{Contact analysis}

The systems of forces presented in Figure 4 reproduce the effect of a homogeneous uniaxial stress on the discrete medium. An eventual effect of the discrete structure may be evaluated by the analysis of vertical and horizontal loading [15, 19]. Considering the symmetries of the cell and the two different loads, we observe only two different types of contact (forces), respectively, identified by the symbols $(\bullet)$ and $(\circ)$.

The normal component $N$ and tangential component $T$ of the contact forces, the normal displacement $\delta_{n}$, and the tangential displacement $\delta_{t}$ of each contact are presented in Table I (for vertical loading) and in Table II (for horizontal loading). The mechanical analysis of the elementary cell is presented in [19].

Two different pairs of contacts are identified in Figure 4: $(\bullet \bullet)$ and $(\bullet \circ)$. Based on the results in Tables I and II, we calculate the values of the mean strains $\bar{\varepsilon}_{n n}$ and $\bar{\varepsilon}_{t t}$ (Equation (2)), the mean stresses $\bar{\sigma}_{n}$ and $\bar{\sigma}_{t}$ (Equation (3)), the principal stresses $\bar{\sigma}_{I}$ and $\bar{\sigma}_{I I}$ (Equation (4) or alternatively (A.6)), and their orientation $\beta$ (Equation (5)) for each pair. All these information is presented in Table III for the vertical loading $P_{y}=\sigma_{y y} d$ and in Table IV for the horizontal loading $P_{x}=\sqrt{3} \sigma_{x x} d$. 

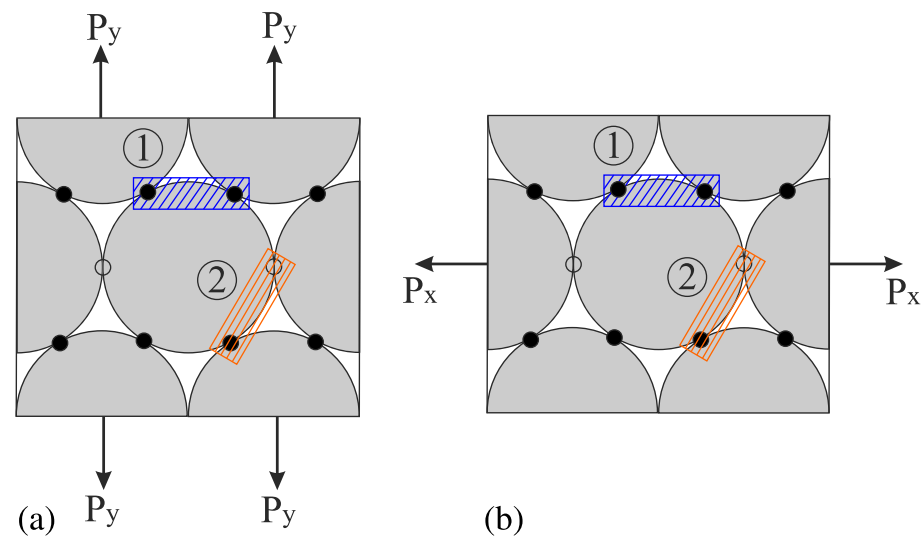

Figure 4. (a) Vertical and (b) horizontal force systems, the two different types of contacts: (•) and (o) and the two different pairs of contacts:

Table I. Contact forces and displacements for uniaxial vertical loading $P_{y}$.

\begin{tabular}{lcccc}
\hline Contacts & $N$ & $T$ & $\delta_{n}$ & $\delta_{t}$ \\
\hline$\bullet$ & $\frac{\sqrt{3}}{6} \frac{2 k_{n}+k_{t}}{k_{n}+k_{t}} P_{y}$ & $\frac{1}{2} \frac{k_{t}}{k_{n}+k_{t}} P_{y}$ & $\frac{\sqrt{3}}{6} \frac{2 k_{n}+k_{t}}{k_{n}\left(k_{n}+k_{t}\right)} P_{y}$ & $\frac{1}{2} \frac{1}{k_{n}+k_{t}} P_{y}$ \\
$\circ$ & $-\frac{\sqrt{3}}{6} \frac{k_{n}-k_{t}}{k_{n}+k_{t}} P_{y}$ & 0 & $-\frac{\sqrt{3}}{6} \frac{k_{n}-k_{t}}{k_{n}\left(k_{n}+k_{t}\right)} P_{y}$ & 0 \\
\hline
\end{tabular}

Table II. Contact forces and displacements for uniaxial horizontal loading $P_{x}$.

\begin{tabular}{ccccc}
\hline Contacts & $N$ & $T$ & $\delta_{n}$ & $\delta_{t}$ \\
\hline$\bullet$ & $\frac{1}{6} \frac{k_{t}}{k_{n}+k_{t}} P_{x}$ & $-\frac{\sqrt{3}}{6} \frac{k_{t}}{k_{n}+k_{t}} P_{x}$ & $\frac{1}{6} \frac{k_{t}}{k_{n}\left(k_{n}+k_{t}\right)} P_{x}$ & $-\frac{\sqrt{3}}{6} \frac{1}{k_{n}+k_{t}} P_{x}$ \\
$\circ$ & $\frac{1}{6} \frac{3 k_{n}+k_{t}}{k_{n}+k_{t}} P_{x}$ & 0 & $\frac{1}{6} \frac{3 k_{n}+k_{t}}{k_{n}\left(k_{n}+k_{t}\right)} P_{x}$ & 0 \\
\hline
\end{tabular}

Table III. Mean strains $\bar{\varepsilon}_{n n}$ and $\bar{\varepsilon}_{t t}$, mean stresses $\bar{\sigma}_{n}$ and $\bar{\sigma}_{t}$, angle $\beta$, and principal stresses $\bar{\sigma}_{I}$ and $\bar{\sigma}_{I I}$ for each pair of contacts for a uniaxial vertical loading $P_{y}=\sigma_{y y} d$.

\begin{tabular}{cccccccc}
\hline Pair & $\bar{\varepsilon}_{n n}$ & $\bar{\varepsilon}_{t t}$ & $\bar{\sigma}_{n}$ & $\bar{\sigma}_{t}$ & $|\beta|$ & $\bar{\sigma}_{I}$ & $\bar{\sigma}_{I I}$ \\
\hline$\bullet$ & $\frac{\sqrt{3}}{6} \frac{3 k_{n}+k_{t}}{k_{n}\left(k_{n}+k_{t}\right) d} P_{y}$ & $-\frac{\sqrt{3}}{6} \frac{k_{n}-k_{t}}{k_{n}\left(k_{n}+k_{t}\right) d} P_{y}$ & $\frac{P_{y}}{d}$ & 0 & 0 & $\sigma_{y y}$ & 0 \\
$\bullet$ & $\frac{\sqrt{3}}{6} \frac{k_{t}}{k_{n}\left(k_{n}+k_{t}\right) d} P_{y}$ & $\frac{\sqrt{3}}{6} \frac{2 k_{n}+k_{t}}{k_{n}\left(k_{n}+k_{t}\right) d} P_{y}$ & $\frac{P_{y}}{4 d}$ & $\frac{\pi}{6}$ & $\frac{\sqrt{3} P_{y}}{4 d}$ & 0 & $\sigma_{y y}$ \\
\hline
\end{tabular}

Table IV. Mean strains $\bar{\varepsilon}_{n n}$ and $\bar{\varepsilon}_{t t}$, mean stresses $\bar{\sigma}_{n}$ and $\bar{\sigma}_{t}$, angle $\beta$, and principal stresses $\bar{\sigma}_{I}$ and $\bar{\sigma}_{I I}$ for each pair of contacts for a uniaxial horizontal loading $P_{x}=$ $\sqrt{3} \sigma_{x x} d$

\begin{tabular}{cccccccc}
\hline Pair & $\bar{\varepsilon}_{n n}$ & $\bar{\varepsilon}_{t t}$ & $\bar{\sigma}_{n}$ & $\bar{\sigma}_{t}$ & $|\beta|$ & $\bar{\sigma}_{I}$ & $\bar{\sigma}_{I I}$ \\
\hline$\bullet \bullet$ & $-\frac{1}{6} \frac{k_{n}-k_{t}}{k_{n}\left(k_{n}+k_{t}\right) d} P_{x}$ & $\frac{1}{6} \frac{3 k_{n}+k_{t}}{k_{n}\left(k_{n}+k_{t}\right) d} P_{x}$ & 0 & 0 & 0 & 0 & $\sigma_{x x}$ \\
$\bullet \circ$ & $\frac{1}{6} \frac{2 k_{n}+k_{t}}{k_{n}\left(k_{n}+k_{t}\right) d} P_{x}$ & $\frac{1}{6} \frac{k_{t}}{k_{n}\left(k_{n}+k_{t}\right) d} P_{x}$ & $\frac{\sqrt{3} P_{x}}{4 d}$ & $\frac{-P_{y}}{4 d}$ & $\frac{\pi}{6}$ & $\sigma_{x x}$ & 0 \\
\hline
\end{tabular}



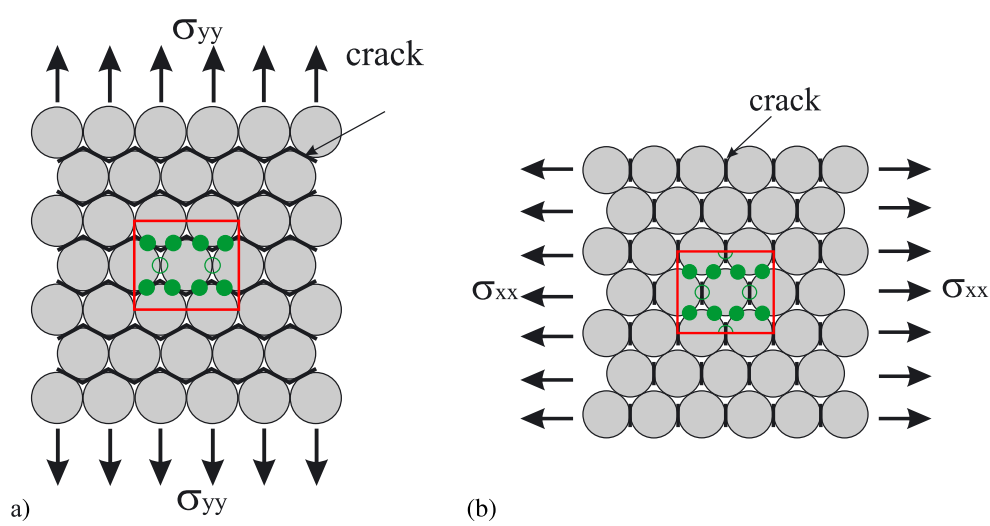

(b)

Figure 5. Expected cracks during a uniaxial tensile rupture: (a) for vertical load and (b) for horizontal load.

\subsection{Tensile strength}

Under homogeneous conditions, we consider the initiation of a quasi-brittle failure when the limit tensile stress $\Sigma^{t}$ is attained for a given direction $\theta_{0}$. At the vicinity of a crack, according to the rupture criterion previously adopted in [15], the failure occurs when the stress intensity factor $K_{\theta \theta}\left(\theta_{0}\right)=\bar{\sigma}_{\theta \theta}\left(\theta_{0}\right) \sqrt{\pi d / 2}$ reaches the value of the material toughness $K_{I C}$ (a polar coordinate system with its origin at the crack tip is considered). In this analysis, the orthoradial stress $\bar{\sigma}_{\theta \theta}\left(\theta_{0}\right)$ is equal to $\bar{\sigma}_{I}$ (for $\beta \leq \pi / 4$ ). For very small cracks (smaller than a particle diameter $d$ ), both conditions can be merged. For a uniaxial loading, from the results of Table III (or Table IV), we deduce the value of the tensile strength:

$$
\Sigma^{t}=K_{I C} \sqrt{\frac{2}{\pi d}}
$$

In the vertical case, as we can see in Table III, only the pairs $(\bullet \bullet)$ are susceptible to break $\left(\bar{\sigma}_{I} \rightarrow \Sigma^{t}\right.$ for $\left.K_{\theta \theta}\left(\theta_{0}\right) \rightarrow K_{I C}\right)$. Both contacts present the same normal force $N$, breaking at the same time (Figure 5(a)). However, on the horizontal case, the highest values of $\bar{\sigma}_{I}$ occurs for the pairs $(\bullet \circ)$. Among them, the contacts $\circ$ present the highest values of the normal force $N$ (as indicated in Table II), which explains their rupture in this case (Figure 5(b)). In any of these configurations, the crack initiation is orthogonal to the direction of the maximum tensile stress, which is physically consistent.

\subsection{Compressive strength}

The cracks preponderantly orthogonal to the compressive direction tends to close (Figure 6(a)), which normally prevents their propagation. Contrariwise, indirect tension may be induced by compression stresses on cracks predominantly parallel to loading (Figure 6(b)). This idea may roughly explain why compressive cracks tends to propagate parallely to the load direction (instead of orthogonally like tensile cracks).

Quasi-brittle failure may also be associated to compressive loading, initiated by the extensive propagation of cracks when the maximum compressive stress supported by the material $\Sigma^{c}$ is reached. In order to characterize the conditions of this failure mode, we propose the evaluation of an apparent stress intensity factor $K_{r r}^{*}\left(\theta_{0}\right)$ :

$$
K_{r r}^{*}\left(\theta_{0}\right)=\bar{\sigma}_{r r}\left(\theta_{0}\right) \sqrt{\frac{\pi d}{2}} .
$$

The direction of the radial stress $\bar{\sigma}_{r r}$ is orthogonal to the direction of the stress $\bar{\sigma}_{\theta \theta}$, consequently it can be simply defined as

$$
\left\{\begin{array}{l}
\bar{\sigma}_{r r}\left(\theta_{0}\right)=\bar{\sigma}_{I I}, \text { if }|\beta| \leq \pi / 4, \\
\bar{\sigma}_{r r}\left(\theta_{0}\right)=\bar{\sigma}_{I}, \text { if }|\beta|>\pi / 4,
\end{array}\right.
$$



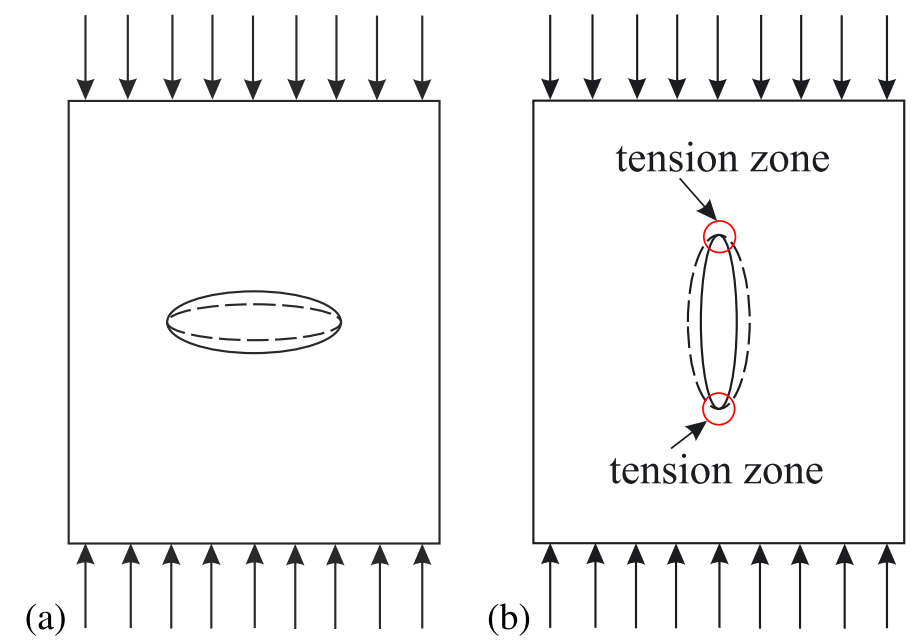

Figure 6. (a) Closing and (b) opening elliptical defects under uniaxial compression.

by analogy to the definition of $\bar{\sigma}_{\theta \theta}\left(\theta_{0}\right)$ [15]. The intensity of the compression is then quantified by the negative value of the apparent stress intensity factor $K_{r r}^{*}\left(\theta_{0}\right)$. A negative stress intensity factor is not directly related to crack propagation (only exceptionally in some cases of micro-buckling of fiber reinforcements under compression, as discussed by Pinho et al. [20]). Hence, we explore this quantity to indirectly characterize this complex mechanism of rupture. A limit for the compressive stress $\Sigma^{c}$ may be simply obtained, similarly to the tensile strength:

$$
\Sigma^{c}=K^{*} \sqrt{\frac{2}{\pi d}}
$$

where we define the (negative) parameter $K^{*}$ as a fictitious toughness. The absence of direct physical meaning of $K^{*}$ (contrarily to the material toughness $K_{I C}$ ) allows us to impose its value as

$$
K^{*}=\Sigma^{c} \sqrt{\frac{\pi d}{2}}
$$

in order to control the compressive strength of the material in our numerical scheme.

On vertical compression (Figure 4(a), for $P_{y}<0$ ), according to Table III, only the pairs $(\bullet \circ)$ are related to an eventual rupture $\left(\bar{\sigma}_{r r}\left(\theta_{0}\right)=\bar{\sigma}_{I I}\right.$, for $\left.\beta \leq \pi / 4\right)$. The contacts (o) are the most tensioned of these pairs (see values of normal forces $N$ on Table I), and therefore, these contacts are broken (Figure 7(a)) when $\bar{\sigma}_{I I} \rightarrow \Sigma^{c}$ (and $K_{r r}^{*}\left(\theta_{0}\right) \rightarrow K^{*}$ ). On horizontal compression (Figure 4(b), for $P_{x}<0$ ), the only candidates to failure are the pairs $(\bullet \bullet)$ as shown on Table IV. When $K_{r r}^{*}\left(\theta_{0}\right) \rightarrow K^{*}$, the two contacts $(\bullet)$ are broken simultaneously (Figure 7(b)). The cracks produced in both directions are thus parallel to the direction of maximum compression, which is physically reasonable.

\subsection{Failure envelope}

The results on Tables III and IV can be superposed (Table V) in the case of a biaxial loading (Figure $8(\mathrm{a})$ ), once the values of $\beta$ are the same for vertical and horizontal uniaxial loading.

The values of the principal stresses $\bar{\sigma}_{I}$ and $\bar{\sigma}_{I I}$ depend individually on $\sigma_{x x}$ or $\sigma_{y y}$, without any combination of them. As previously discussed (Sections 3.2 and 3.3), the failure depends on $\bar{\sigma}_{I}$ in tension and depends on $\bar{\sigma}_{I I}$ in compression. If we impose $\Sigma^{t}$ as an upper bound for $\bar{\sigma}_{I}$ and $\Sigma^{c}$ as a lower bound for $\bar{\sigma}_{I I}$, we obtain the Rankine's criterion (Figure 8(b)) as the failure envelope. 

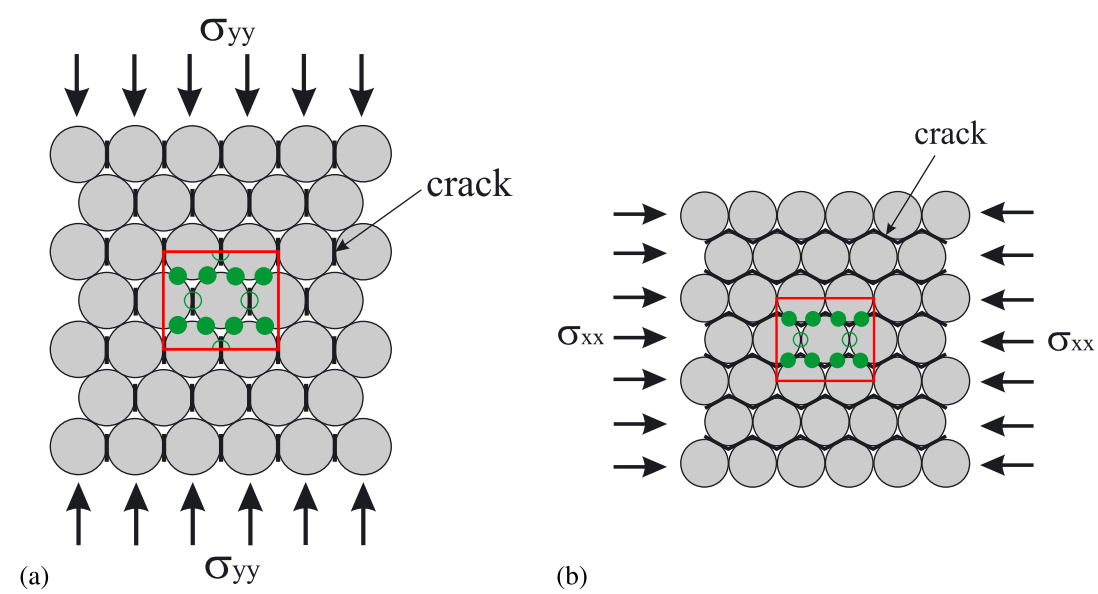

(b)

Figure 7. Expected cracks during a uniaxial compressive rupture: (a) for vertical load and (b) for horizontal load.

Table V. Principal stresses $\bar{\sigma}_{I}$ and $\bar{\sigma}_{I I}$ for each pair of contacts under biaxial loading.

\begin{tabular}{ccc}
\hline Pair & $\bar{\sigma}_{I}$ & $\bar{\sigma}_{I I}$ \\
\hline$\bullet \bullet$ & $\sigma_{y y}$ & $\sigma_{x x}$ \\
$\bullet \bullet$ & $\sigma_{x x}$ & $\sigma_{y y}$ \\
\hline
\end{tabular}
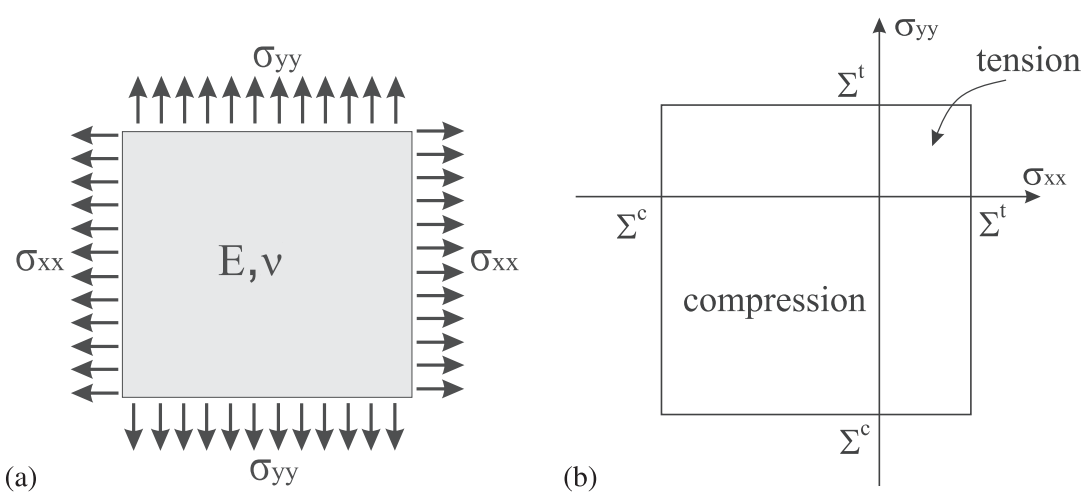

Figure 8. (a) Biaxial stress state and (b) the failure envelope of Rankine's criterion.

\section{NUMERICAL TESTS}

\subsection{Biaxial stress rupture}

We compare the predictions of Section 3 with respect to the strength of materials under biaxial stresses to discrete element simulations. The tests are performed on square samples of dimension $L$, composed by particles of diameter $d$. Three samples with different ratio $L / d$ are presented: 44,88 , and 176.

The stresses are increased until failure with a constant ratio $\phi=\sigma_{y y} / \sigma_{x x}$. Because of the homogeneous stress conditions, the samples present a generalized rupture, with a large number of contact broken for a given limit loading. We verify the rupture envelope testing various values of $\phi$. The system of units is the same presented in [15], where the stress scale is given by the ratio 

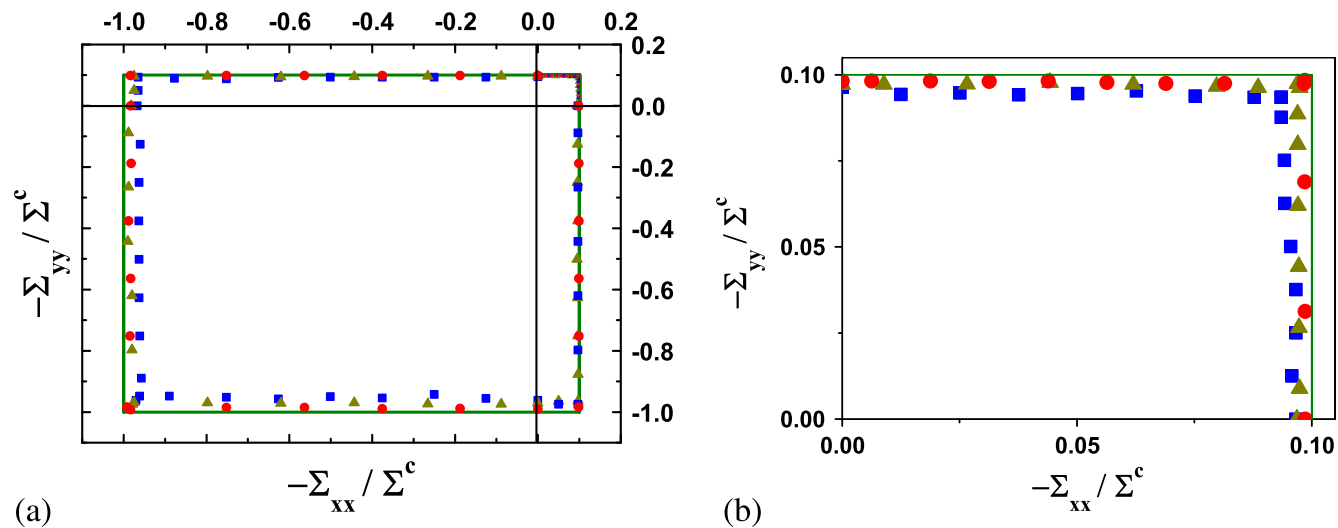

Figure 9. (a) Failure envelope for $\Sigma^{c} / \Sigma^{t}=-10$ and (b) zoom on the tensile behavior for various samples: (ם) $L / d=44,(\Delta) L / d=88,(\bullet) L / d=176$.

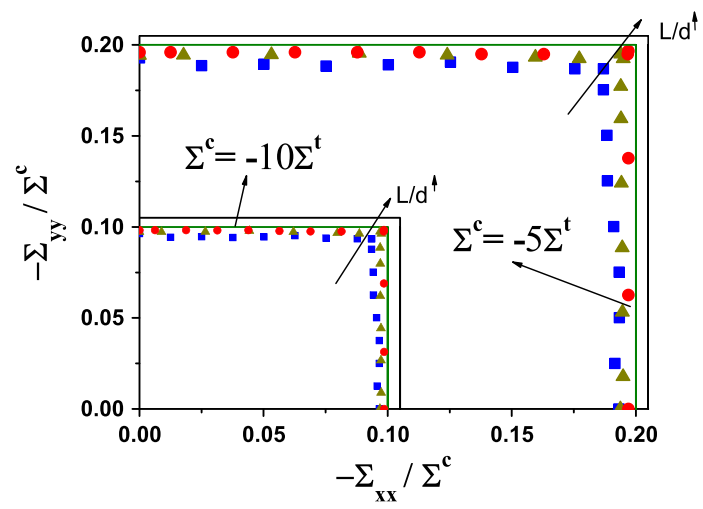

Figure 10. Biaxial tensile behavior of two different materials with $\Sigma^{c} / \Sigma^{t}=-10$ and $\Sigma^{c} / \Sigma^{t}=-5$, respectively, for various samples: (ם) $L / d=44,(\Delta) L / d=88,(\bullet) L / d=176$.

between toughness and particle diameter $K_{I C} / \sqrt{d}$. Defined values of toughness and particle diameter impose the tensile strength $\Sigma^{t}=K_{I C} \sqrt{2 /(\pi d)}$ to the material (Equation (6)). The compressive strength $\Sigma^{c}$ is independently defined by the value of the fictitious toughness $K^{*}$ (Equation (9)).

A very good agreement is shown by the comparison between numerical results and the theoretical Rankine-like behavior (Figure 9) for $\Sigma^{c} / \Sigma^{t}=-10\left(K^{*}=-10 K_{I C}\right)$.

In Figure 10, we compare the results for materials with different ratios between compressive and tensile strengths: $\Sigma^{c} / \Sigma^{t}=-10$ and $\Sigma^{c} / \Sigma^{t}=-5$. Given that the stresses are normalized here by $\Sigma^{c}$, we focus on the tensile behavior.

The verifications indicate that tensile and compressive strengths are precisely controlled in our model. The small deviations of the expected behaviors are mostly due to boundary effects caused by the moving rigid walls used to apply the stress states. These effects tend to vanish away when the ratio $L / d$ is increased, as shown on Figure 10 and discussed in [19].

\subsection{Bending fracture}

The quasi-brittle failure of a three-point bending beam (Figure 11) usually occurs because of a local rupture in its middle section, giving rise to a vertical crack. Hence, this setup is specially useful to analyze crack initiation and its propagation.

The beam is composed by a quasi-brittle material of Young's modulus $E=30 G P a$, Poisson's ratio $v=0.2$, toughness $K_{I C}=1 M P a \sqrt{m}$, tensile strength $\Sigma^{t}=8 M P a$, and compressive strength $\Sigma^{c}=-80 M P a$. The dimensions of the beam are indicated in Figure 11. 


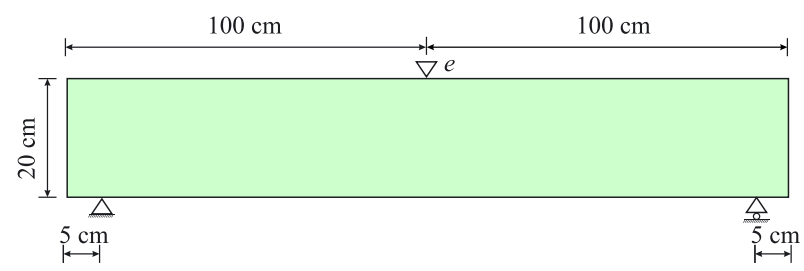

Figure 11. Configuration of the three-point bending test.

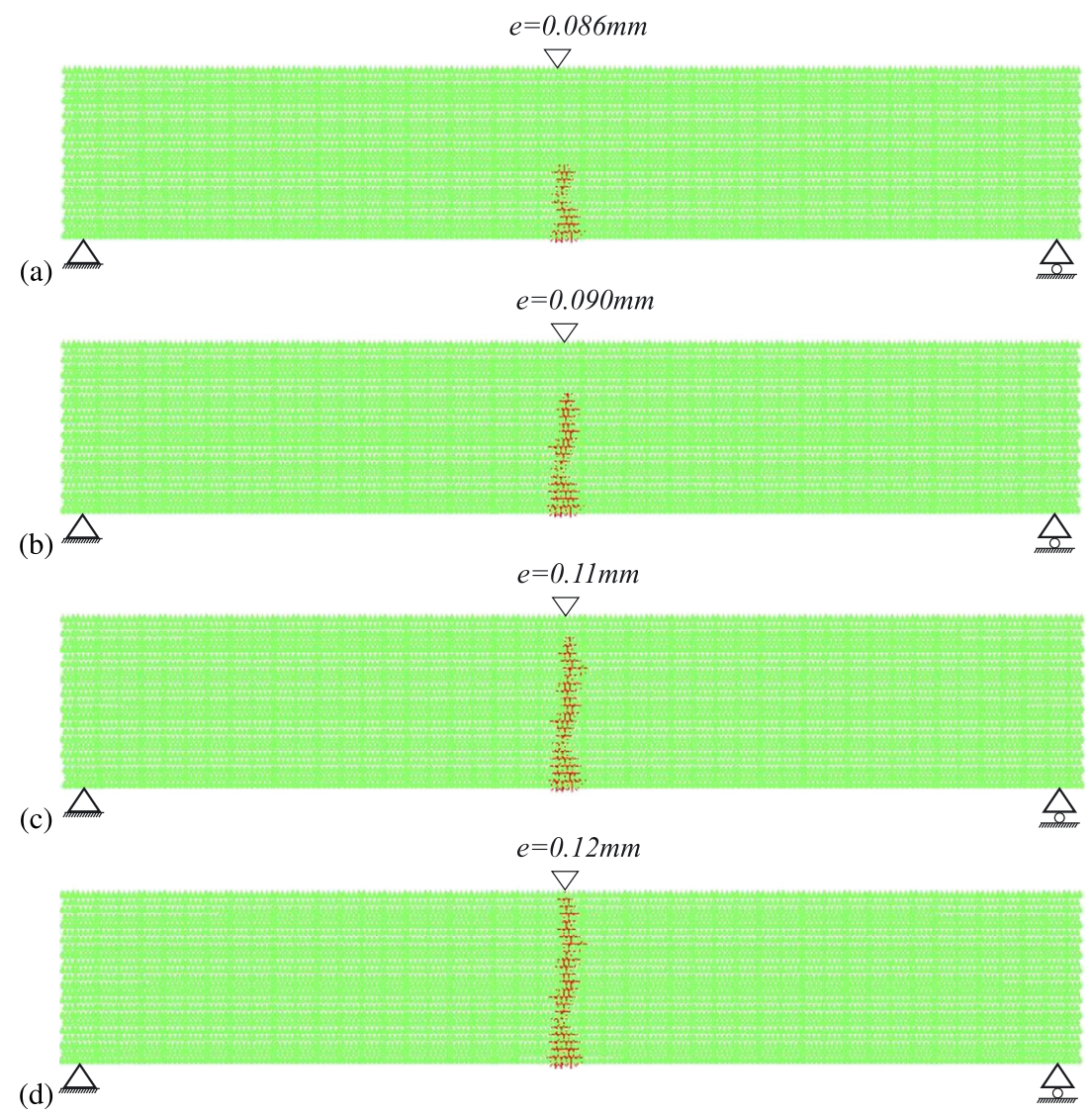

Figure 12. Crack propagation during a three-point bending test at different imposed displacements (a) $e=$ $0.086 \mathrm{~mm}$, (b) $e=0.090 \mathrm{~mm}$, (c) $e=0.11 \mathrm{~mm}$, and (d) $e=0.12 \mathrm{~mm}$.

A particle diameter $d=1 \mathrm{~cm}$ associates the correct relationship between the toughness and the tensile strength (Equation (6)); the fictitious toughness $K^{*}=-10 K_{I C}$ sets the coherent ratio between the compressive and tensile strengths $\Sigma^{c} / \Sigma^{t}=-10$. The sample presents 4589 particles. The displacement imposed by the central support induces a maximum normal stress at the center of the beam (tension on the bottom and compression on the top). Considering that the tensile strength is 10 times lower than the compressive strength, a tensile crack initiation is observed at the bottom of the middle section, orthogonally to the axis of the beam. The expected vertical propagation of the crack is presented in Figure 12 for increasing displacements $e$ of the central support.

\subsection{Diametral compression fracture}

The diametral compression test is widely used to measure the tensile strength of quasi-brittle materials. As indicated in Figure 13(a), the compressive force (per unit of thickness) $P$ induces a tensile stress $\sigma=2 P /(\pi D)$ perpendicular to the loaded diameter $D$. 
(a)

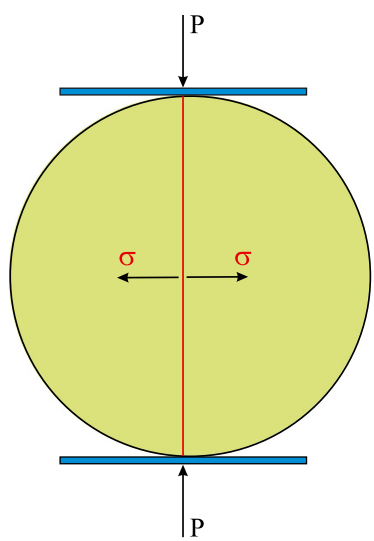

(b)

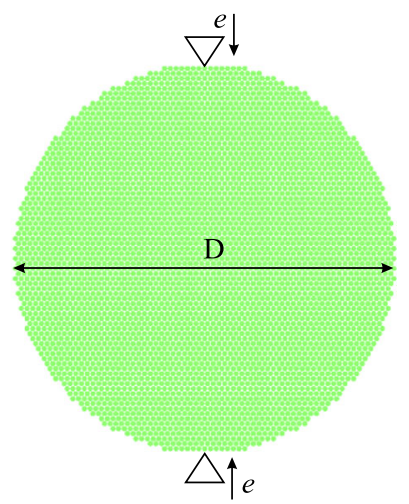

Figure 13. (a) Diametral compression test and induced tensile stress. (b) Corresponding discrete element model.

(a)

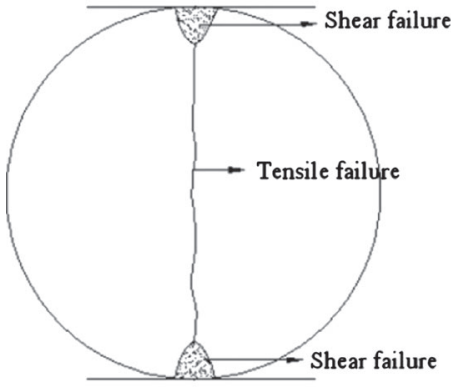

(b)

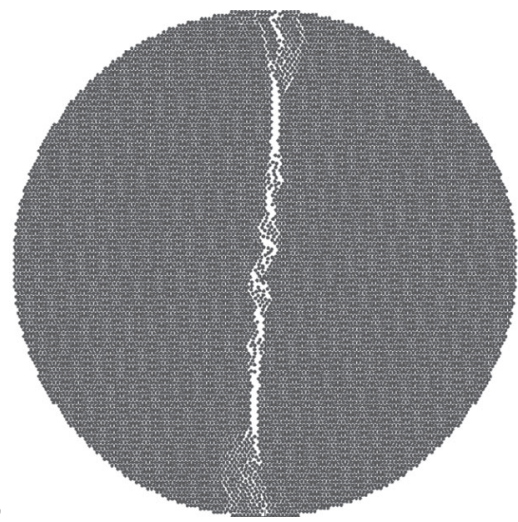

Figure 14. (a) Transition between shear and tensile failure modes in a diametral compression test, modified from the illustration by $[21,22]$. (b) Simulation result of a diametral compression test for $\Sigma^{c}=-50 \Sigma^{t}$.

The aim of our simulations is to analyze the rupture pattern and its consistency with the material strength. Our sample (of diameter $D=66 \mathrm{~mm}$ ) is composed of a material of Young's modulus $E=60 G P a$, Poisson's ratio $v=0.23$, and toughness $K_{I C}=1 M P a \sqrt{m}$. We choose a particle diameter $d=0.5 \mathrm{~mm}$, which associates a tensile strength $\Sigma^{t}=35.7 M P a$. The samples present 15,847 particles. The force $P$ is induced by the imposed displacement $e$ as indicated in Figure 13(b).

For the first test, we adopt a high compressive strength $\Sigma^{c}=-50 \Sigma^{t}\left(K^{*}=-50 K_{I C}\right)$ to decrease the effect of crushing at the loading points, intending to model an ideal indirect tensile test. The location where the maximum tensile strain occurs is usually described as corresponding to a failure mode transition between shear failure and tensile failure (Figure 14(a)), as observed for many rock types in the diametral compression test [21]. The simulation seems to capture these two mechanisms as indicated in Figure 14(b). The central splitting fracture line should be associated with a tensile failure mode. However, it is difficult to judge whether the tensile fracture initiates and propagates to the center or from the center during experimental study [22]. In this numerical test, the tensile crack propagated along the diameter towards the center of the specimen.

In the second test, we intended to observe the consequences on the rupture pattern of a lower compressive strength $\Sigma^{c}=-5 \Sigma^{t}$. For materials with low compression-tension ratios, the failure may occur away from the center of the specimen, and the obtained value of the tensile strength is lower than the true value $[22,23]$. The flaws that are activated in the initial loading stages dominate the fracturing rather than the general stress distribution. En echelon patterns (step-like features), often associated with compressive stress fields, can be observed in discs subjected to diametral compression tests and in numerical simulations [24] as shown in Figure 15(a). The mechanism of 
(a)

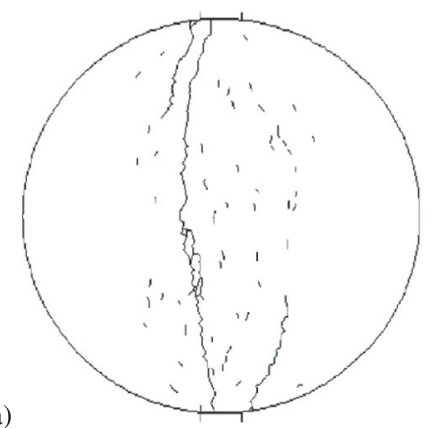

(b)

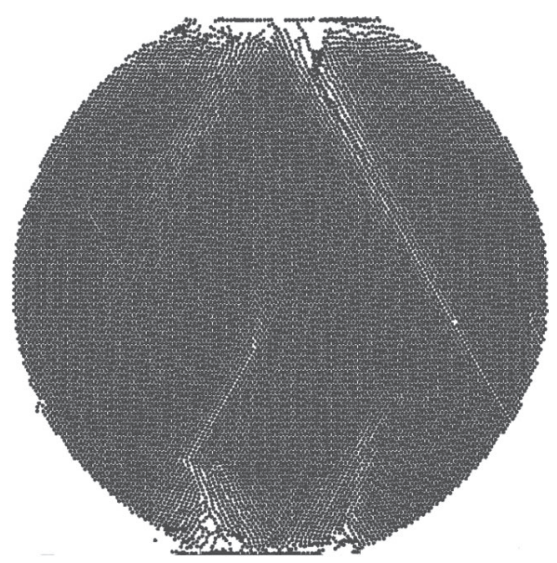

Figure 15. (a) Fracture path forming an en echelon pattern [24] in a diametral compression test. (b) Simulation result of a diametral compression test for $\Sigma^{c}=-5 \Sigma^{t}$.

compressive failure proposed in Section 3.3 induces a realistic deviation of the crack path from the diameter of the sample (Figure 15(b)). However, we observe inclined rupture bands instead of individual cracks because of the homogeneity of the material. After large loading displacements $e$, a loss of the symmetry of the rupture pattern is associated with the propagation of cracks with privileged directions. It suggests an effect of the particle structure for large strains.

\section{CONCLUSIONS}

A recurrent difficulty on discrete element models is the complex identification of contact parameters, which demands calibration procedures to identify the best choice to approximate a given material behavior. In our present model, the tensile strength is expressed as a clear function of the toughness and the diameter of the particles, simplifying considerably the material description. Furthermore, compressive strength becomes a direct (and completely independent) parameter of the model. This original feature may be specially useful in some fields (i.e., rock mechanics), where compressive strength is often much higher than tensile strength (which are hardly simulated with usual contact models).

Theoretical predictions are confirmed by simulations of classical systems. A Rankine-like failure envelope is verified for various square samples under different biaxial stress combinations. The initiation and the propagation of cracks are analyzed based on three-point bending and diametral compression tests. In all examples, consistent results are observed concerning the failure loading and propagation patterns.

Finally, the proposed approach intended to improve the control of the material properties and its homogeneity in DEM simulations. Nevertheless, the mechanical behavior may be strongly affected by the disorder in materials [25-27]. The effect of discontinuities, flaws, and heterogeneities (precisely introduced at the contact level) is an important element for the realistic description of the complex failure mechanisms of geomaterials.

\section{APPENDIX A: DETERMINATION OF $\beta$ AND $\bar{\sigma}_{I I}$ FOR A PAIR OF CONTACTS}

The value of $\beta$ depends on the values of mean strains and mean stresses at a pair of contacts. The mean strains $\bar{\varepsilon}_{n n}$ and $\bar{\varepsilon}_{t t}$ are related to the mean principal strains $\bar{\varepsilon}_{I}$ and $\bar{\varepsilon}_{I I}$ (considering the coordinate system on Figure 3) by

$$
\left\{\begin{array}{l}
\bar{\varepsilon}_{n n}=\bar{\varepsilon}_{I} \cos ^{2} \beta+\bar{\varepsilon}_{I I} \sin ^{2} \beta, \\
\bar{\varepsilon}_{t t}=\bar{\varepsilon}_{I} \sin ^{2} \beta+\bar{\varepsilon}_{I I} \cos ^{2} \beta .
\end{array}\right.
$$


For a homogeneous material, the elastic behavior can be written as a function of Young's modulus $E$ and Poisson's ratio $v$. Hence, the principal stresses are directly related to principal strains (in two dimensions) by the expression:

$$
\left\{\begin{array}{l}
\bar{\varepsilon}_{I}=\frac{1+v}{E} \bar{\sigma}_{I}-\frac{v}{E}\left(\bar{\sigma}_{I}+\bar{\sigma}_{I I}\right), \\
\bar{\varepsilon}_{I I}=\frac{1+v}{E} \bar{\sigma}_{I I}-\frac{v}{E}\left(\bar{\sigma}_{I}+\bar{\sigma}_{I I}\right) .
\end{array}\right.
$$

As indicated by Equation (A.1) and (A.2), the trace of the strain tensor $\left(\bar{\varepsilon}_{n n}+\bar{\varepsilon}_{t t}=\bar{\varepsilon}_{I}+\bar{\varepsilon}_{I I}\right)$ is related to the trace of the stress tensor by

$$
\bar{\varepsilon}_{n n}+\bar{\varepsilon}_{t t}=\frac{1-v}{E}\left(\bar{\sigma}_{I}+\bar{\sigma}_{I I}\right)
$$

If we substitute the mean principal stresses (Equation (4)) into Equation (A.3), isolating $\beta$, we obtain simply

$$
\tan \beta-\frac{1}{\tan \beta}=-\frac{2}{\tan (2 \beta)}=\frac{A}{\bar{\sigma}_{t}}
$$

where $A=\frac{E}{1-v}\left(\bar{\varepsilon}_{n n}+\bar{\varepsilon}_{t t}\right)-2 \bar{\sigma}_{n}$. Finally, the value of $\beta$ can be written as

$$
\beta=-\frac{1}{2} \arctan \left(\frac{2 \bar{\sigma}_{t}}{A}\right)
$$

The expression of the second principal stress $\bar{\sigma}_{I I}$ on Equation (4) is sensitive to small values of $\beta$. It can be alternatively calculated (Equation (A.3)) as a function of the mean strains $\bar{\varepsilon}_{n n}$ and $\bar{\varepsilon}_{t t}$ and the value of $\sigma_{I}$ :

$$
\bar{\sigma}_{I I}=\frac{E}{1-v}\left(\bar{\varepsilon}_{n n}+\bar{\varepsilon}_{t t}\right)-\bar{\sigma}_{I}
$$

\section{REFERENCES}

1. Bazant ZP, Cedolin L. Blunt crack band propagation in finite element analysis. Journal of the Engineering Mechanics Division 1979; 105:297-315.

2. Bazant ZP. Instability, ductility and size effect in strain softening concrete. Journal of the Engineering Mechanics Division 1976; 102:331-344.

3. Zhang J, Li XB. A failure criterion for brittle and quasi-brittle materials under any level of stress concentration. Engineering Fracture Mechanics 2008; 75:4925-4932.

4. Novozhilov V. On a necessary and sufficient criterion for brittle strength. Journal of Applied Mathematics and Mechanics; 147:212-222, 33(2).

5. Leguillon D. A critetion for crack nucleation at a notch in homogeneous materials. Comptes Rendus de l'Académie des Sciences - Series IIB - Mechanics 2001; 329:97-102.

6. Leguillon D. Strength or toughness? A criterion for crack onset at a notch. European Journal of Mechanics - A/Solids 2002; 21:61-72.

7. Cundall PA, Strack ODL. A discrete numerical model for granular assemblies. Géotechnique 1979; 29:47-65.

8. Donzé F, Mora P, Magnier SA. Numerical simulation of faults and shear zones. Geophysical Journal International 1994; 116:46-52.

9. Ergenzinger C, Seifried R, Eberhard P. A discrete element model to describe failure of strong rock in uniaxial compression. Granular Matter 2010; 13:341-364.

10. Matsuda Y, Iwase Y. Numerical simulation of rock fracture using three-dimensional extended discrete element method. Earth Planets Space 2002; 54:367-378.

11. Donzé F, Magnier SA. Numerical simulations of impacts using a discrete element method. Mechanics of CohesiveFrictional Materials 1998; 3:257-276.

12. Cundall PA, Lee CA, Potyondy DO. Modeling rock using bonded assemblies of circular particles. Proceedings of the 2nd N. American Rock Mechanics Symposium, Montreal, 1996; 1937-1944.

13. Potyondi DO, Cundall PA. A bonded-particle model for rock. International Journal of Rock Mechanics and Mining Sciences 2004; 41(8):1329-1364.

14. Liu C, Pollard DD, Shi B. Analytical solutions and numerical tests of elastic and failure behaviors of close-packed lattice for brittle rocks and crystals. Journal of Geophysical Research 2013; 118(1):71-82. 
15. Le BD, Koval G, Chazallon C. Discrete element model for crack propagation in brittle materials. International Journal for Numerical and Analytical Methods in Geomechanics 2016; 40(4):583-595.

16. Koval G, Roux J-N, Corfdir A, Chevoir F. Annular shear of cohesionless granular materials: From the inertial to quasistatic regime. Physical Review E 2009; 79(2):021306.

17. Allen MP, Tildesley DJ. Computer Simulation of Liquids. Oxford University Press: Oxford, 1987.

18. Tavarez FA, Plesha ME. Discrete element method for modelling solid and particulate materials. International Journal for Numerical Methods in Engineering 2007; 70:379-404.

19. Le BD, Koval G, Chazallon C. Discrete element approach in brittle fracture mechanics. Engineering Computations 2013; 30(2):263-276.

20. Pinho ST, Robinson P, Iannucci L. Fracture toughness of the tensile and compressive fibre failure modes in laminated composites. Composites Science and Technology 2006; 66(13):2069-2079.

21. Hobbs DW. The tensile strength of rocks. International Journal of Rock Mechanics and Mining Sciences 1964; 1(3):385-396.

22. Li D, Wong LNY. The brazilian disc test for rock mechanics applications: review and new insights. Rock Mechanics and Rock Engineering 2013; 46(2):269-287.

23. Fairhurst C. On the validity of the 'Brazilian' test for brittle materials. International Journal of Rock Mechanics and Mining Sciences \& Geomechanics Abstracts 1964; 1(4):535-546.

24. Steen BVD, Vervoort A, Napier JAL. Observed and simulated fracture pattern in diametrically loaded discs of rock material. International Journal of Fracture 2005; 131(1):35-52.

25. Pompe W, Herrmann HJ, Roux S. Statistical models for the fracture of disordered media. North-Holland, 1990, $353 \mathrm{p.}$ Crystal Research and Technology 1991; 26(8):1076-1076.

26. Alava MJ, Nukala PKVV, Zapperi S. Statistical models of fracture. Advances in Physics; 55(3-4):349-476.

27. Ostoja-Starzewski M. Microstructural Randomness and Scaling in Mechanics of Materials. Chapman and Hall/CRC, 2007. 